\title{
Real-World Experience With a Tapered Biodegradable Polymer-Coated Sirolimus-Eluting Stent in Patients With Long Coronary Artery Stenoses
}

\author{
Alessandro Lupi ${ }^{\mathrm{a}, \mathrm{d}}$, Fabrizio $\mathrm{Ugo}^{\mathrm{b}}{ }^{\text {, Leonardo De Martino }}{ }^{\mathrm{a}}$, Vincenzo Infantino ${ }^{\mathrm{c}}$, Mario Iannaccone ${ }^{\mathrm{b}}$, \\ Sergio Iorio ${ }^{\mathrm{a}}$, Angelo Di $\mathrm{Leo}^{\mathrm{c}}$, Salvatore Colangelo ${ }^{\mathrm{b}}$, Marco Zanera ${ }^{\mathrm{c}}$, Alon Schaffer ${ }^{\mathrm{a}}$, \\ Simone Persampieri ${ }^{\mathrm{a}}$, Roberto Garbo $^{\mathrm{b}}$, Gaetano Senatore ${ }^{\mathrm{c}}$
}

\begin{abstract}
Background: Treatment of long coronary stenoses (LCS) with long tapered drug-eluting stents (LT-DES) would offer clinical and economic benefits. However, the feasibility of an interventional strategy based upon the systematic LCS treatment with an LT-DES has not been evaluated so far.

Methods: We performed a multicenter prospective study including consecutive patients with: 1) An LCS $>25 \mathrm{~mm}$ at coronary angiography; 2) An attempt to fix the LCS with a single BioMime Morph ${ }^{\mathrm{TM}}$ stent, a novel LT-DES available from 30 to $60 \mathrm{~mm}$ long. The primary efficacy endpoint was procedural success. The secondary safety endpoints were post-procedural TIMI3 flow, stent detachment during delivery, acute stent thrombosis and in-hospital mortality.
\end{abstract}

Results: From February 2017 to March 2018, we recorded 272 patients with an LCS and an attempt to deploy an LT-DES during percutaneous coronary intervention $(\mathrm{PCI})(69.3 \pm 11.4$ years, $75.7 \%$ males, $25.7 \%$ diabetic and $43.8 \%$ with acute coronary syndromes, mean LCS length 48.8 $\pm 9.5 \mathrm{~mm}$ ). LT-DES deployment was successful in 262 patients $(96.3 \%)$, and failure occurred without stent detachment or other complications. Final TIMI3 flow was present in $270(99.3 \%)$ patients. In-hospital death occurred in five patients $(1.8 \%)$, with no case of acute stent thrombosis, recurrent myocardial infarction or repeated revascularization.

Conclusion: In this real-world study, a strategy of fixing LCS with a single LT-DES was feasible and safe, with a high rate of procedural success and a low rate of in-hospital complications. More extensive randomized studies are warranted to assess the potential clinical and economic benefits of LT-DES.

Manuscript submitted March 29, 2020, accepted April 20, 2020

Published online June 3, 2020

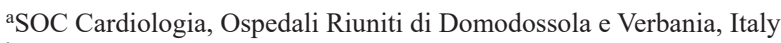
bDepartment of Invasive Cardiology, Ospedale San Giovanni Bosco, Turin, Italy 'Department of Cardiology, Ospedale Civile, Cirie, Italy

${ }^{\mathrm{d} C o r r e s p o n d i n g ~ A u t h o r: ~ A l e s s a n d r o ~ L u p i, ~ S O C ~ C a r d i o l o g i a, ~ O s p e d a l i ~ R i u n i t i ~}$ di Domossola e Verbania, ASL V.C.O., Via Mazzini 117, 28887 Omegna (VB), Italy. Email: lupialessandro1@gmail.com

doi: https://doi.org/10.14740/cr1055
Keywords: Long coronary lesions; Biodegradable polymer; Tapered stents; Overlapping stents; Complex coronary interventions

\section{Introduction}

In patients with coronary artery disease (CAD), aging and comorbidities contribute to the complexity of coronary lesions [1, 2]. Long, tortuous and calcified coronary stenoses represent, more and more frequently, the daily challenge for the interventional cardiologist $[3,4]$. In particular, very long coronary stenoses (LCS) may need more than one stent for full lesion coverage. Consequent strut overlapping is a well-recognized trigger for stent thrombosis and restenosis $[5,6]$. Moreover, coronary vessels taper between proximal and distal segments. Significant diameter discrepancies may favor a "two-stent strategy" and, in such cases, the stent overlapping is unavoidable. Furthermore, "spot stenting" in long and diffuse CAD predisposes to geographical miss, avoidable by using long stents. Finally, a "long stent strategy" has the potential to decrease stent-related costs.

BioMime Morph ${ }^{\mathrm{TM}}$ (BM) (Meril Life Sciences Pvt. Ltd, Gujarat, India) is a new tapered drug-eluting stent (DES), available up to $60 \mathrm{~mm}$ of length. Therefore, this stent is particularly suitable for interventional strategies aiming to respect the physiological tapering of native coronaries, to avoid DES overlapping or geographical miss and to abate procedural DES-related costs.

In the present study, we evaluated the feasibility and safety of an interventional strategy based upon the employ long tapered DES (LT-DES) to fix LCS. To this end, we prospectively assessed, on an intention to treat basis, a cohort of consecutive "all-comers" CAD patients with a single long coronary stenotic lesion treated in our institutions with a single BM stent.

\section{Materials and Methods}

\section{Device description and interventional procedure}

BM uses the NexGen ${ }^{\mathrm{TM}}$ ultrathin $(65 \mu \mathrm{m})$ cobalt-chromium tapered platform with a cell design mixing open and close cells 
to maintain resistance to longitudinal deformation. BM coating is a biodegradable thin copolymer formulation combining PLL and PLGA, which acts as a carrier for sirolimus, loaded with a dose of $1.25 \mu \mathrm{g} / \mathrm{mm} 2$ of stent surface area. The stent is available in sizes up to $60 \mathrm{~mm}$ lengths and tapered diameters up to $4.00 / 3.50 \mathrm{~mm}$.

Patients received medications according to usual practice and percutaneous coronary intervention (PCI) was performed using standard techniques [7]. Since the first availability of BM in Italy, the cath labs involved in the present study adopted a common interventional strategy attempting BM deployment in patients with LCS. Stenoses $\geq 25 \mathrm{~mm}$ at quantitative coronary angiography (QCA, Xcelera ${ }^{\circledR}$ measurement software, Philips Medical Systems, NE) were considered suitable for BM implantation. The final choice to deploy a BM was any way at the operator discretion, as well as the prescription of procedural antithrombotic therapy.

\section{Study design and population}

The present investigation was a prospective, observational, multicenter study conducted in three high-volume Italian catheterization laboratories (St. Giovanni Bosco Hospital - Turin, Civil Hospital - Cirie and St. Biagio Hospital - Domodossola).

Inclusion criteria were: 1) Angiographic demonstration of a single LCS $\geq 25 \mathrm{~mm}$, according to QCA; 2) An attempt during PCI to treat the LCS with a single BM stent.

Exclusion criteria were: 1) Age $<18$ years; 2) Presence of more than one LCS; 3) LCS classified as chronic total occlusions with a J-CTO score $\geq 3$ [8]. Data from consecutive patients fulfilling these inclusion and exclusion criteria were retrospectively collected from local clinical databases and pooled together for statistical analysis, on an intention-to-treat basis.

\section{Endpoints definitions}

We considered as primary endpoint the angiographically successful deployment of BM stent (residual stenosis $<20 \%$ and TIMI flow grade 3 in the treated vessel).

Secondary endpoints were the rates of: 1) Use of a buddy wire or a "child in mother" devices to advance the stent to the target lesion; 2) Stent detachment from the balloon during delivery; 3) Acute stent thrombosis, according to the Academic Research Consortium (ARC) classification [9]; 4) Postprocedural TIMI3 flow grade; 5) All-cause in-hospital death; 6) Non-fatal in-hospital myocardial infarction; 7) In-hospital urgent repeated revascularization.

\section{Statistical analysis}

We present categorical data as frequencies (percentages of the total). We tested for normal distribution datasets with continuous variables using the Shapiro-Wilk normality test. In the presence of normal distribution, we present data as mean values \pm standard deviation (SD), otherwise, as median values with interquartile range $(25-75 \%)$.

We also performed a stepwise logistic regression analysis to search for independent predictors of procedural complications by comparing significantly different clinical and angiographic features in patients with successful and unsuccessful stent implantation. We entered en-bloc into the model each covariate associated with successful stent deployment at a significance level of $\mathrm{P}<0.1$. We tested potential interactions between covariates, excluding those affected by multicollinearity. We finally reported results as correlation coefficients and tested the final model with the Hosmer-Lemeshow goodness-of-fit test.

The occurrence of a two-sided $\mathrm{P}$ value of less than 0.05 was considered statistically significant. To perform the statistical analysis, we used the Statistical Package for Social Sciences, version 18 (SPSS Inc., Chicago, IL, USA).

\section{Ethical issues}

The study fulfilled the Local Institutional Review Board guidelines for observational research and conformed with the principles of the Declaration of Helsinki [10].

\section{Results}

\section{Baseline, angiographic and procedural characteristics}

From February 2017, the date of the first availability for clinical practice of BM in our country, to March 2018, a total of 272 patients fulfilled the inclusion and exclusion criteria of the study. The mean age of the cohort was $69.3 \pm 11.4$ years, $75.7 \%$ of the patients were males, $75.0 \%$ were hypertensive, $25.7 \%$ had type 2 diabetes and $43.8 \%$ suffered from an acute coronary syndrome (ACS). Table 1 reports the baseline demographic and clinical characteristics of the study cohort.

Mean coronary lesion length was $48.8 \pm 9.5 \mathrm{~mm}, 50.0 \%$ of the lesions were in the left anterior descending artery and 97.8\% were Ellis type B2 or C according to American College of Cardiology/American Heart Association (ACC/AHA) classification. The transradial route was employed in $87.9 \%$ of patients and a switch from radial to femoral access to complete the BM deployment was never needed. Table 2 summarizes the angiographic and procedural details.

\section{Intraprocedural outcomes}

Table 2 reports the interventional procedure characteristics and Table 3 summarizes antithrombotic therapy of the study population. BM deployment was successful in 262 patients (96.7\%). No case of accidental stent detachment occurred during target lesion crossing and, in each case of BM deployment failure, the operators switched to a "two-stent strategy" without further complications. Successful BM deployment required a buddy wire in 49 patients $(18.0 \%)$, a "child in mother" device (GuideLinerTM, Vascular Solutions Inc., Minneapolis, $\mathrm{MN}, \mathrm{USA})$ in 14 patients $(5.2 \%)$ and an anchoring balloon in 


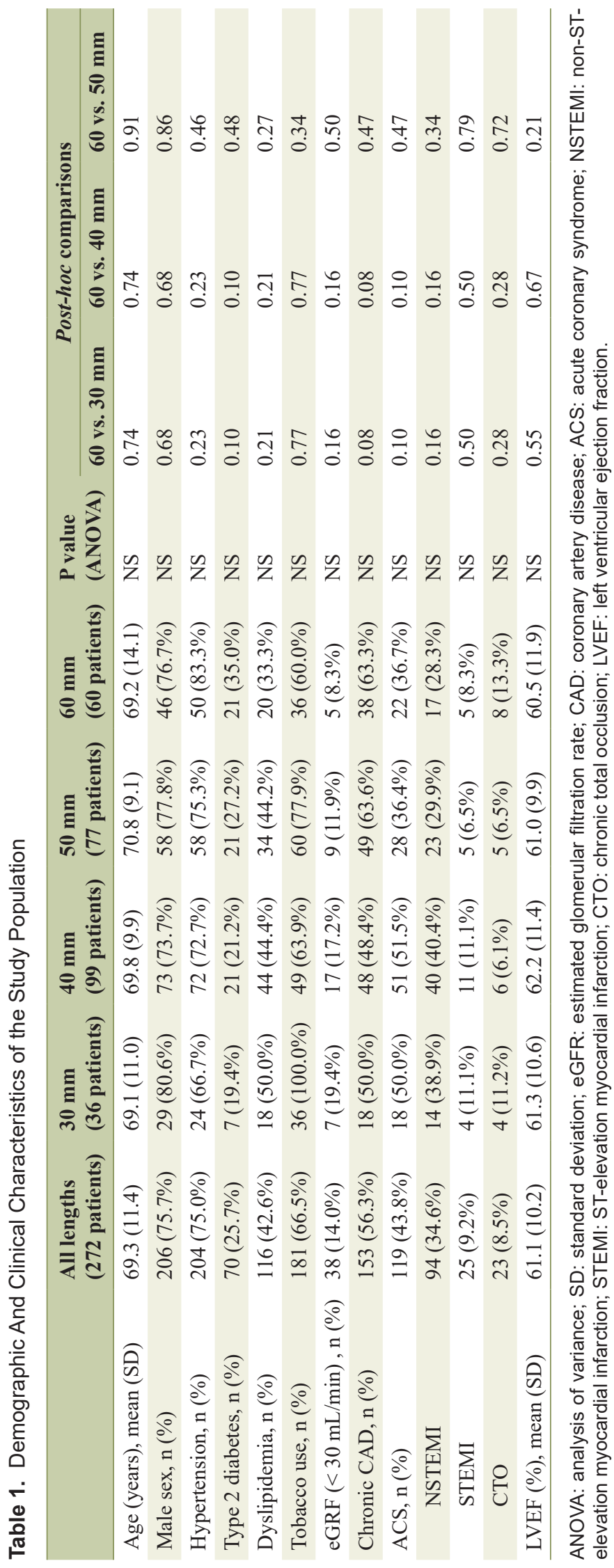

seven patients (2.6\%). Postprocedural TIMI3 flow occurred in the vast majority of patients $(99.3 \%)$. The arterial access was trans-radial in 239 patients $(87.9 \%)$, and no switch from radial to femoral access was needed to achieve the successful BM deployment.

Logistic regression analysis identified age $(\mathrm{P}=0.017)$, severe coronary artery tortuosity $(\mathrm{P}=0.005)$ and stenosis length $(\mathrm{P}=0.006)$ as the only independent predictors of BM deployment failure (Table 4).

\section{In-hospital outcomes}

In-hospital death occurred in five patients $(1.8 \%)$, three formerly admitted for ACS complicated by refractory congestive heart failure and two for non-cardiac causes. During the hospital stay, no patient suffered from stent thrombosis, recurrent nonfatal myocardial infarction or repeated revascularization of the coronary vessel treated with the BM stent.

\section{Analysis according to stent length}

The study patients, grouped according to BM length (30, 40, 50 and $60 \mathrm{~mm}$ ), showed no statistical difference in the main clinical features (Table 1). Patients treated with the $60 \mathrm{~mm}$ $\mathrm{BM}$ presented more diseased vessels per patient in comparison with those treated with 30 and $40 \mathrm{~mm} \mathrm{BM}(2.2 \pm 0.7 \mathrm{vs}$. $1.8 \pm 0.8$ and $1.7 \pm 0.7$, respectively; $\mathrm{P}=0.001$ for both comparisons, Table 2), while we observed no significant difference in comparison with patients treated with the $50 \mathrm{~mm} \mathrm{BM}(2.2$ \pm 0.7 vs. $1.9 \pm 0.7, \mathrm{P}=0.16$, Table 2 ). As expected, patients treated with the $60 \mathrm{~mm}$ stent showed longer stenotic coronary segments in comparison with those treated with 30,40 and 50 $\mathrm{mm}$ BM $(61.5 \pm 6.1 \mathrm{~mm}$ vs. $29.9 \pm 6.4 \mathrm{~mm}, 38.2 \pm 7.6 \mathrm{~mm}$ and $51.6 \pm 7.5 \mathrm{~mm}, \mathrm{P}<0.0001$ for each comparison, Table 2 ), but BM length did not influence procedural outcomes in most cases (Table 2). A child-in-mother device was used more frequently in patients treated with $60 \mathrm{~mm}$ in comparison with those treated with 30 or $40 \mathrm{~mm} \mathrm{BM}(11.7 \%$ vs. $0.0 \%, \mathrm{P}=0.04$ and $3.0 \%, P=0.03$ respectively, Table 2 ). Finally, the need for a buddy-wire was more frequent in patients treated with the 60 $\mathrm{mm} \mathrm{BM}$, in comparison with those treated with the $50 \mathrm{~mm} \mathrm{BM}$ $(30.0 \%$ vs. $11.7 \%, \mathrm{P}=0.007)$.

\section{Discussion}

In our study, the strategy to treat very LCS with LT-DES demonstrated excellent profiles of efficacy and safety, even in a "real-world" setting. The study included patients with type 2 diabetes, ACS and complex CAD, representing real clinical practice. BM deployment was successful in the vast majority of them, even if interventions often required the support of a buddy wire, a "child-in-mother" device or an anchoring balloon. Age, severe tortuosity and length of the treated coronary vessel were, as expected, independent predictors of BM deployment failure. However, LT-DES use was remarkably safe, as the oc- 


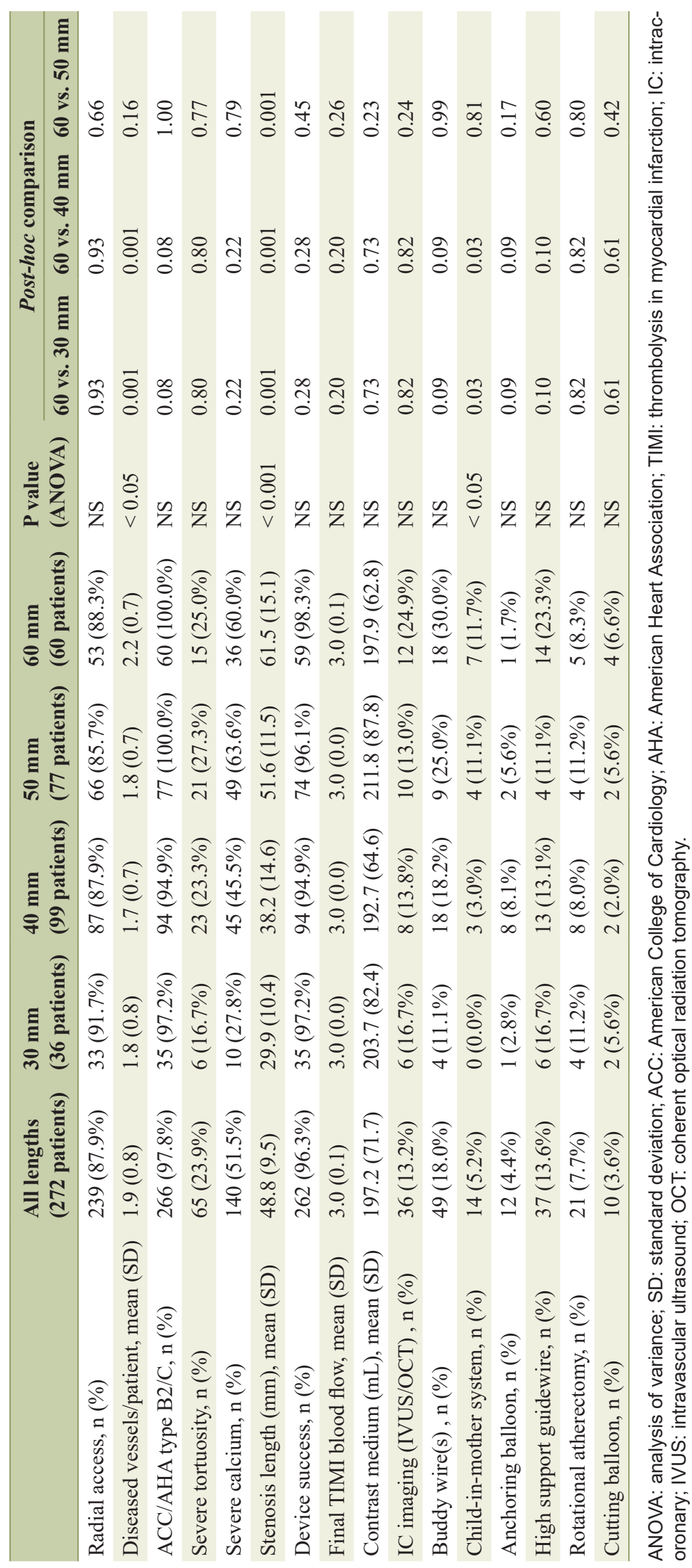


Table 3. Antithrombotic Treatment Before, During and Following PCI

\begin{tabular}{lllll}
\hline \multirow{2}{*}{ Antithrombotic treatments } & \multicolumn{3}{c}{ Antithrombotic treatment start } \\
\cline { 2 - 5 } Clopidogrel & Pre-PCI, n (\%) & Peri-PCI and post-PCI, n (\%) & Discharge, n (\%) \\
\hline Ticagrelor & $31(11.4 \%)$ & $156(57.4 \%)$ & $192(70.6 \%)$ & $187(68.8 \%)$ \\
Prasugrel & $70(25.7 \%)$ & $5(1.8 \%)$ & $68(25.0 \%)$ & $75(27.6 \%)$ \\
Aspirin & $10(3.7 \%)$ & $0(0.0 \%)$ & $12(4.4 \%)$ & $10(3.7 \%)$ \\
Dual antiplatelet therapy & $195(71.7 \%)$ & $61(22.4 \%)$ & $256(94.1 \%)$ & $256(94.1 \%)$ \\
Unfractionated heparin & $111(40.8 \%)$ & $145(53.3 \%)$ & $256(94.1 \%)$ & $256(94.1 \%)$ \\
Bivalirudin & $0(0.0 \%)$ & $153(56.3 \%)$ & $0(0.0 \%)$ & $153(56.3 \%)$ \\
Enoxaparin & $0(0.0 \%)$ & $4(1.5 \%)$ & $0(0.0 \%)$ & $4(1.5 \%)$ \\
Warfarin & $115(42.3 \%)$ & $0(0.0 \%)$ & $0(0.0 \%)$ & $2(0.7 \%)$ \\
Dabigatran & $6(2.2 \%)$ & $2(0.7 \%)$ & $115(42.3 \%)$ & $8(2.9 \%)$ \\
Oral AntiXa & $2(0.7 \%)$ & $16(5.9 \%)$ & $6(2.2 \%)$ & $10(3.7 \%)$ \\
\hline
\end{tabular}

PCl: percutaneous cardiovascular intervention.

casional inability to deploy the stent never compromised the procedure, completed in all these patients by switching to a "two-stent" strategy. Moreover, neither stent detachment nor in-hospital stent thrombosis complicated BM deployment attempts. Of note, transradial PCI procedures were almost $90 \%$ and switching from radial to femoral access to complete the stent deployment was never needed.

The BM shares with the well-studied BioMime ${ }^{\mathrm{TM}}$ DES technological characteristic (sirolimus elution, biodegradable polymeric carrier and ultrathin stent struts) associated with low late lumen loss and reduced hazard of thrombus formation in both studies [11-15] and meta-analyses [16, 17]. The primary safety and efficacy trials meriT-1 [18] and meriT-2 [19] and, more recently, the observational post-marketing multisite registry meriT-3 [14] reported low rates of major adverse cardiovascular events (MACEs) and late stent thrombosis. Finally, the randomized meriT-5 trial [20] demonstrated that BM was non-inferior to the XIENCE DES at 9-month follow-up.
The added value of the BM stent is the availability of very long stent measures and the tapered design, aimed at respecting the anatomy of coronary vessels. These innovative characteristics are particularly useful according to the following considerations. Firstly, age and comorbidities rates are increasing in CAD patients treated in our catheterization laboratories. This fact reflects in increasing complexity of coronary lesions $[1,2]$. Secondly, long, tortuous and heavily calcified coronary lesions generally require overlapping DES, a known trigger for thrombosis and restenosis $[5,6]$. Third, treating LCS with conventional long stents is challenging, as coronary vessels physiologically taper between proximal and distal segments. This situation often forces interventional cardiologists to a "two-stent strategy", to avoid stent undersizing and coronary overstretching or rupture. Fourth, diffuse CAD predisposes to geographical miss with a "spot-stent" strategy, while long stents are potentially less affected by stent misplacement and remaining gaps. Finally,

Table 4. Predictors of Successful BioMime Morph Stent Deployment According to Logistic Regression Analysis

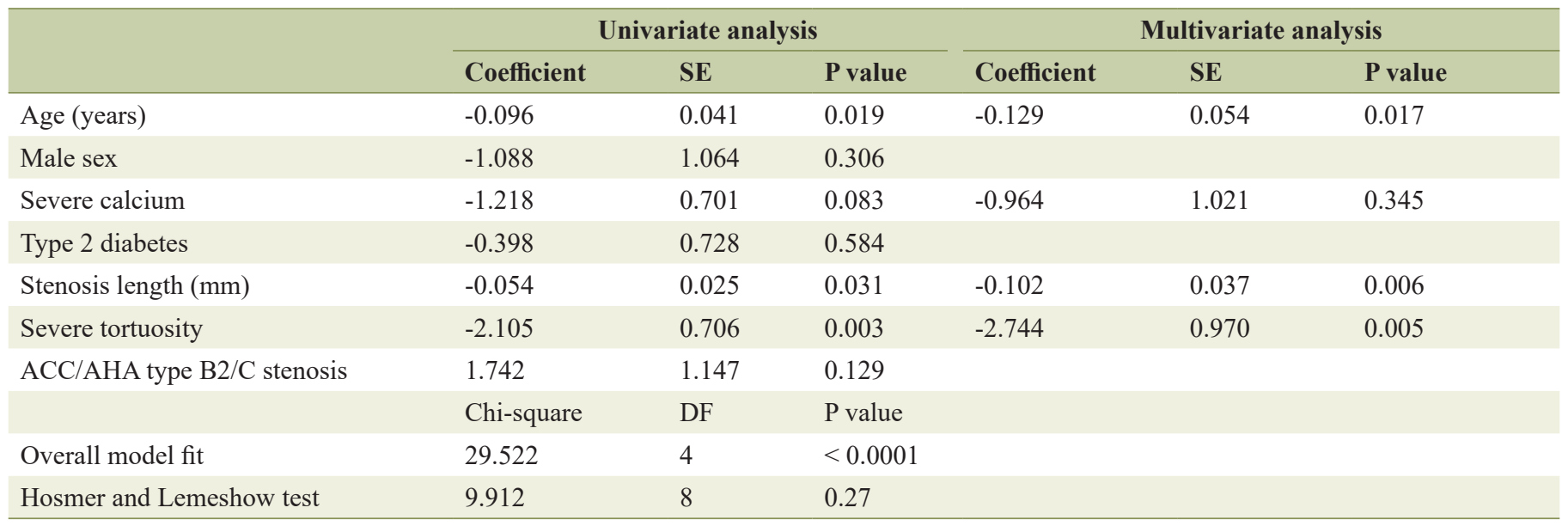

SE: standard error; DF: degrees of freedom; ACC: American College of Cardiology; AHA: American Heart Association. 
a long stent strategy might abate procedural costs, reducing significantly the number of stents needed to fix long diseased coronary segments.

The novel BM combines in the same device tapered geometry, low profile, good trackability and the availability of very long measures, up to $60 \mathrm{~mm}$. Thus, BM is particularly suitable for interventional strategies aiming to respect the physiological tapering of native coronaries, to avoid DES overlapping or geographical miss and to decrease procedural DES-related costs.

Data about the interventional performance and clinical results of this new device are limited to some case reports [21, 22] and a recent study by Valero et al describing the procedural outcomes of $50 \mathrm{CAD}$ patients treated with the $60 \mathrm{~mm}$ BM [23]. These authors reported a 92\% rate of successful BM deployment, with $18 \%$ of GuideLiner ${ }^{\mathrm{TM}}$ use to deploy the stent. In this small cohort, no MACE was observed over a 275 days median follow-up. These figures are substantially similar to those emerging from our study, indicating that BM has the potential to achieve the same results even if used in different countries and by different physicians with different interventional skills. Finally, a recent systematic review and network meta-analysis of contemporary randomized controlled trials comparing optimal medical therapy (OMT), coronary artery bypass grafting and different stent types in stable CAD demonstrated that, compared to OMT, none of the stent types included was associated with a lower risk of death. However, durable-polymer-CoCr-everolimus DES and bioabsorbable-polymer-CoCrsirolimus DES, like BM, were associated with a lower risk of myocardial infarction than OMT, adding pieces of evidence to the advantages of the BM platform [24].

\section{Study limitations}

Our study has the well-known limitations of registries and observational studies. The absence of randomization exposes our results to selection bias, even if partially mitigated by the consecutive enrolment of patients fulfilling the inclusion criteria of the study. Moreover, the relatively small number of patients studied and the limitation of follow-up to the in-hospital period should prevent us from drawing firm conclusions about "hard" endpoints like mortality or stent thrombosis. Finally, the confirmation of the presence of a coronary stenotic lesion $\geq 25$ $\mathrm{mm}$ relied upon QCA. The choice of the better projection to minimize coronary segment foreshortening and the selection of the distal and proximal markers of the coronary stenoses are operator-dependent and thus a potential source of additional biases.

\section{Conclusions}

In a "real-world" setting, treating LCS systematically with an LT-DES appears feasible, with promising interventional performance and safety profiles. However, more extensive randomized studies with a longer follow-up are necessary to compare LT-DES with multiple overlapping conventional DES in treating very long and diffuse coronary artery lesions.

\section{Acknowledgments}

We are grateful to the Research Nurses staff of the Cardiology Division for their precious support to our study.

\section{Financial Disclosure}

The study was not supported by any public or private grant/ funding.

\section{Conflict of Interest}

All the authors declare no known conflict of interest to disclose.

\section{Informed Consent}

At the moment of hospital admission, each patient included in the study signed informed consent to the employ of his/her medical documentation for research/study reasons.

\section{Author Contributions}

Alessandro Lupi: study design, data analysis, manuscript drafting, statistical analysis. Fabrizio Ugo: manuscript drafting. Leonardo De Martino: manuscript drafting. Vincenzo Infantino: manuscript drafting. Mario Iannaccone: study design, data analysis, manuscript drafting, statistical analysis. Sergio Iorio: manuscript drafting. Angelo Di Leo: manuscript drafting. Salvatore Colangelo: manuscript drafting. Marco Zanera: manuscript drafting. Alon Schaffer: manuscript drafting. Simone Persampieri: data analysis, manuscript drafting. Roberto Garbo: manuscript drafting. Gaetano Senatore: manuscript drafting.

\section{Data Availability}

The data supporting the findings of this study are available from the corresponding author upon reasonable request. Any inquiries regarding supporting data availability of this study should be directed to the corresponding author.

\section{References}

1. Go AS, Mozaffarian D, Roger VL, Benjamin EJ, Berry JD, Borden WB, Bravata DM, et al. Heart disease and stroke statistics - 2013 update: a report from the American Heart Association. Circulation. 2013;127(1):e6-e245.

2. Newman AB, Naydeck B, Sutton-Tyrrell K, Edmundowicz D, Gottdiener J, Kuller LH. Coronary artery calcification in older adults with minimal clinical or subclinical cardiovascular disease. J Am Geriatr Soc. 2000;48(3):256-263. 
3. Kobayashi Y, Okura H, Kume T, Yamada R, Kobayashi Y, Fukuhara K, Koyama T, et al. Impact of target lesion coronary calcification on stent expansion. Circ J. 2014;78(9):2209-2214.

4. Iannaccone M, Barbero U, D'Ascenzo F, Latib A, Pennacchi M, Rossi ML, Ugo F, et al. Rotational atherectomy in very long lesions: Results for the ROTATE registry. Catheter Cardiovasc Interv. 2016;88(6):E164-E172.

5. Raber L, Juni P, Loffel L, Wandel S, Cook S, Wenaweser $\mathrm{P}$, Togni M, et al. Impact of stent overlap on angiographic and long-term clinical outcome in patients undergoing drug-eluting stent implantation. J Am Coll Cardiol. 2010;55(12):1178-1188.

6. Mori N, Okamoto N, Tanaka A, Yano M, Makino N, Egami Y, Shutta R, et al. Comparison of angiographic and 1 -year outcomes between a long single stent and overlapping double stents in patients with newer-generation drug-eluting stents for long narrowings. Am J Cardiol. 2016;117(11):1724-1728.

7. Authors/Task Force $\mathrm{m}$, Windecker S, Kolh P, Alfonso F, Collet JP, Cremer J, Falk V, et al. 2014 ESC/EACTS Guidelines on myocardial revascularization: The Task Force on Myocardial Revascularization of the European Society of Cardiology (ESC) and the European Association for Cardio-Thoracic Surgery (EACTS)Developed with the special contribution of the European Association of Percutaneous Cardiovascular Interventions (EAPCI). Eur Heart J. 2014;35(37):2541-2619.

8. Morino Y, Abe M, Morimoto T, Kimura T, Hayashi Y, Muramatsu T, Ochiai M, et al. Predicting successful guidewire crossing through chronic total occlusion of native coronary lesions within 30 minutes: the J-CTO (Multicenter CTO Registry in Japan) score as a difficulty grading and time assessment tool. JACC Cardiovasc Interv. 2011;4(2):213-221.

9. Mauri L, Hsieh WH, Massaro JM, Ho KK, D'Agostino R, Cutlip DE. Stent thrombosis in randomized clinical trials of drug-eluting stents. N Engl J Med. 2007;356(10):10201029.

10. Rits IA. Declaration of Helsinki. Recommendations guidings doctors in clinical research. World Med J. 1964;11:281.

11. Natsuaki M, Kozuma K, Morimoto T, Kadota K, Muramatsu T, Nakagawa Y, Akasaka T, et al. Biodegradable polymer biolimus-eluting stent versus durable polymer everolimus-eluting stent: a randomized, controlled, noninferiority trial. J Am Coll Cardiol. 2013;62(3):181-190.

12. Danzi GB, Chevalier B, Urban P, Fath-Ordoubadi F, Carrie $\mathrm{D}$, Wiemer $\mathrm{M}$, Serra A, et al. Clinical performance of a drug-eluting stent with a biodegradable polymer in an unselected patient population: the NOBORI 2 study. EuroIntervention. 2012;8(1):109-116.

13. Mehta AB, Chandra P, Dalal J, Shetty P, Desai D, Chocklingam K, Prajapati J, et al. One-year clinical outcomes of BioMatrix-Biolimus A9 eluting stent: the e-BioMatrix multicenter post marketing surveillance registry in India. Indian Heart J. 2013;65(5):593-599.

14. Jain RK, Chakravarthi P, Shetty R, Ramchandra P, Polavarapu RS, Wander GS, Mohan B, et al. One-year outcomes of a BioMime Sirolimus-Eluting Coronary Stent
System with a biodegradable polymer in all-comers coronary artery disease patients: The meriT-3 study. Indian Heart J. 2016;68(5):599-603.

15. Windecker S, Haude M, Neumann FJ, Stangl K, Witzenbichler B, Slagboom T, Sabate M, et al. Comparison of a novel biodegradable polymer sirolimus-eluting stent with a durable polymer everolimus-eluting stent: results of the randomized BIOFLOW-II trial. Circ Cardiovasc Interv. 2015;8(2):e001441.

16. Lupi A, Gabrio Secco G, Rognoni A, Lazzero M, Fattori $\mathrm{R}$, Sheiban I, Sante Bongo A, et al. Meta-analysis of bioabsorbable versus durable polymer drug-eluting stents in 20,005 patients with coronary artery disease: an update. Catheter Cardiovasc Interv. 2014;83(6):E193-206.

17. Bangalore S, Toklu B, Patel N, Feit F, Stone GW. Newer-Generation Ultrathin Strut Drug-Eluting Stents Versus Older Second-Generation Thicker Strut DrugEluting Stents for Coronary Artery Disease. Circulation. 2018;138(20):2216-2226.

18. Dani S, Costa RA, Joshi H, Shah J, Pandya R, Virmani $\mathrm{R}$, Sheiban I, et al. First-in-human evaluation of the novel BioMime sirolimus-eluting coronary stent with bioabsorbable polymer for the treatment of single de novo lesions located in native coronary vessels - results from the meriT-1 trial. EuroIntervention. 2013;9(4):493-500.

19. Seth A CR, Kaul U, Wander GS, Mullasari A, Nanjappa MC, Heggunje-Shetty P, Alexander T, et al. Late angiographic and clinical outcomes of the novel BioMimeTM sirolimus-eluting coronary stent with ultra-thin cobaltchromium platform and biodegradable polymer for the treatment of diseased coronary vessels: results from the prospective, multicentre meriT-2 clinical trial. Asia Intervention. 2016;2:19-27.

20. Abizaid A, Kedev S, Kedhi E, Talwar S, Erglis A, Hlinomaz O, Masotti M, et al. Randomised comparison of a biodegradable polymer ultra-thin sirolimus-eluting stent versus a durable polymer everolimus-eluting stent in patients with de novo native coronary artery lesions: the meriT-V trial. EuroIntervention. 2018;14(11):e1207e1214.

21. Matchin YG, Atanesyan RV, Kononets EN, Danilov NM, Bubnov DS, Ageev FT. [The first experience of using very long stents covered with sirolimus $(4060 \mathrm{~mm})$ in the treatment of patients with extensive and diffuse lesions of the coronary arteries]. Kardiologiia. 2017;57(4):19-26.

22. Sinha SK, Mahrotra A, Abhishekh NK, Razi M, Aggarwal P, Tripathi S, Rekwaal L, et al. Acute stent loss and its retrieval of a long, tapering morph stent in a tortuous, calcified lesion. Cardiol Res. 2018;9(1):63-67.

23. Valero E, Consuegra-Sanchez L, Minana G, Garcia-Blas S, Rodriguez JC, Moyano P, Sanchis J, et al. Initial experience with the novel BioMime $60 \mathrm{~mm}$-long sirolimuseluting tapered stent system in long coronary lesions. EuroIntervention. 2018;13(13):1591-1594.

24. Taglieri N, Bruno AG, Bacchi Reggiani ML, D'Angelo EC, Ghetti G, Bruno M, Palmerini T, et al. Impact of coronary bypass or stenting on mortality and myocardial infarction in stable coronary artery disease. Int J Cardiol. 2020;309:63-69. 